Cuestiones de filosofía

ISSN: 0123-5095

Vol. 2 - No. 18

Enero - junio, año 2016

pp. $185-214$

\title{
LA FORMACIÓN INTEGRAL: Una apuesta de la educación superior ${ }^{1}$
}

\author{
INTEGRAL FORMATION: \\ A bet of higher education
}

\author{
Adriana Judith Nova-Herrera ${ }^{2}$ \\ Fundación Universitaria Juan de Castellanos, Colombia
}

Fecha de recepción: 23 de noviembre de 2015

Concepto de evaluación: 13 de febrero de 2016

Fecha de aprobación: 22 de junio de 2016

\section{Resumen}

Con base en las leyes 30 de 1992 y 115 de 1994, la misión de la educación superior en Colombia es formar integralmente al ser humano; esta tarea es asumida por las instituciones de educación superior, pero, a diferencia de la investigación y los saberes técnicos, es difícil identificarla en la práctica. $\mathrm{La}$ formación humana de Kant y Locke fundamentan la formación integral, y la visión contemporánea de esta se apoya en los planteamientos de Rincón, Orozco, Campo y Restrepo y Zazar, quienes afirman que el fin de la educación es llevar al ser a alcanzar su humanización, a partir del desarrollo de su condición y naturaleza, para lograr formar personas plenamente desarrolladas y sociedades más justas y pacíficas. Pero a la educación superior se le delegan múltiples responsabilidades que la desvían de su fin. Este artículo presenta una revisión

\footnotetext{
${ }^{1}$ El escrito se deriva de los avances del proyecto de tesis doctoral «Límites y alcances de la formación integral en la UPTC 2008-2010», adscrito al grupo RELIGIO.

${ }^{2}$ Administradora de Empresas, Magistra en Educación, Estudiante Doctorado en Ciencias de la Educación. Coordinadora del Centro de Investigación y Extensión de la Facultad de Ciencias de la Educación y Humanidades de la Fundación Universitaria Juan de Castellanos, Tunja, correo electrónico: adrisnova@yahoo.com.ar.
} 
de teorías que se enmarcan desde el período de la Ilustración hasta finales del siglo XX, junto con algunos documentos gubernamentales. Este proceso se realizó a través de una conceptualización teórica, asumida como una primera fase del análisis de contenido cualitativo que se aplica en el proyecto «Límites y alcances de la formación integral en la UPTC. Sede Tunja 2008-2010». El presente documento evidencia la necesidad de una visión de educación superior más integradora. Se destaca la importancia de incluir las corrientes humanistas en la relación enseñanza-aprendizaje.

Palabras clave: Formación. Formación integral. Educación superior.

\begin{abstract}
Providing integral formation to human beings is the higher education mission in Colombia, based on Laws 115 of 1994 and 30 of 1992. This task is undertaken for the higher education institutions; however, unlike research and the technical knowledge, the comprehensive training is difficult to be identified in practice. Human education in Kant and Locke support the comprehensive training; while that contemporary vision is based in the proposals of Rincón, Orozco, Campo $\&$ Restrepo, and Zazar, who claim that the goal of the higher education, is to lead the human being to achieve his humanization, acording her or his condition and nature development; in order to get the persons formation fully developed and societies more just and peaceful.

But to the higher education are delegated many responsibilities that move it away from its mission. This paper presents a revision of theories from the Enlightenment to the end of the XX century, plus some governmental documents. This process made across a theoretical conceptualization, is the first step of the qualitative content analysis, applied on the project «Límites y Alcances de la Formación Integral en la UPTC. Sede Tunja 2008-2010». This document evidences the higher education need for a more inclusive vision. It highlights the humanist trends inclusion, inside the teaching-learning relationship's importance.
\end{abstract}

Keywords: Integral Formation. Comprehensive Training. Higher Education. 


\section{INTRODUCCIÓN}

La educación, a lo largo de la historia de la humanidad, ha sido vista como una herramienta esencial para construir la sociedad deseada. Bien lo anunció Delors: «Frente a los numerosos desafíos del porvenir, la educación constituye un instrumento indispensable para que la humanidad pueda progresar hacia los ideales de paz, libertad y justicia social» (1996, p. 13). Amedida que la economía, la tecnología, la cultura, la ciencia y la comunicación han presentado cambios, la educación ha tenido que replantear sus intereses de formación; sin embargo, la formación de la condición humana que propone la formación integral es considerada la esencia que articula todas las acciones educativas, para alcanzar el ideal de ciudadanos comprometidos con la construcción de una mejor sociedad.

Las instituciones de educación superior, así como las de educación preescolar, básica y media, también deben brindar una formación integral; este compromiso es recordado desde los aspectos misionales de los centros educativos: su papel en la sociedad es «formar profesionales íntegros». Esto implica desarrollar habilidades, conocimientos y actitudes en la persona, de manera que logre la autonomía e independencia (Echeverría, 2010) para tomar decisiones responsables con su propia vida, con los demás y con todo lo que le rodea.

Del profesional se espera que transforme la sociedad para mejorar las condiciones de vida; es por esto que se hace imperante atender las necesidades de desarrollo espiritual, al igual que las cognitivas. Algunos autores dicen que en el mundo de hoy se está viviendo una crisis social causada por las dinámicas de los mercados mundiales, el capitalismo, el interés económico y el consumismo (Nussbaum, 2010; Huanacuni, 2010). Los pueblos indígenas están haciendo un llamado al «vivir bien», como prioridad fundamental de la humanidad; pues los discursos occidentales -especialmente- para lograr una supuesta «mejor calidad de vida» están llevando a la insensibilización, a la infelicidad, a la soledad, al individualismo, a la destrucción humana y de la madre tierra (Huanacuni, 2010).

Bajo esta situación, se propone la tesis doctoral «Límites y alcances de la formación integral en la Universidad Pedagógica y Tecnológica de ColombiaUPTC-, Sede Tunja. 2008-2010». Dentro de ella se plantea el objetivo de determinar los límites y alcances de la formación integral de los estudiantes que 
ingresaron entre los años 2008 y 2010 a los pregrados presenciales en la sede Tunja, a través de sus experiencias vividas y la de sus profesores, con miras a reconocer el papel de la universidad como agente que participa en la formación de la condición humana de sus estudiantes.

En este artículo se presenta la primera etapa del desarrollo de la investigación, que giró en torno a responder las preguntas: ¿Cuáles son los antecedentes de la formación integral?, ¿cuál es el concepto de la formación integral? y ¿cuáles son los fines de la educación superior en la sociedad actual, caracterizada por el interés de alcanzar un desarrollo económico? Se parte, entonces, de la construcción de la conceptualización teórica, que soportará el análisis de contenido de documentos institucionales que contienen una política de formación en la educación superior a nivel supranacional, nacional e institucional, para posteriormente construir el instrumento de recolección de información que permita reconocer las experiencias de estudiantes y docentes.

Para el desarrollo de la conceptualización que propone la herramienta de análisis de contenido, método de la investigación, se tomaron los temas centrales de la propuesta doctoral. Se inicia por reconocer en la historia de la educación la consolidación de un pensamiento sobre la formación orientada a desarrollar el crecimiento como ser humano. Una educación así, acentuada en los aspectos humanos, se encuentra especialmente en las ideas de Locke y Kant, filósofos clásicos de la educación, seguramente inspirados en antiguos pensadores. Si bien sus ideas de formación no eran para todos, en razón a la visión del mundo que se tenía en Occidente en los siglos XVII y XVIII, concentrada en una educación para la burguesía masculina, estos postulados se convirtieron en la base del pensamiento educativo humanista.

En la segunda parte se analiza el concepto de formación consolidado en los siglos XX y XXI. Se da inicio al recorrido teórico desde la hermenéutica de Gadamer (1960/1999), dado que, para comprender el método de las ciencias del espíritu, explica los conceptos básicos del humanismo: formación del hombre, Sensus Communis (sentido común) y la capacidad de juicio. En su primer apartado, Gadamer hace una exposición de lo que significa «formación» y establece un fundamento esencial para la acción educativa de hoy.

Estos postulados de Gadamer se complementan con la visión de autores contemporáneos que definen la formación como eje central de la educación. 
La mayoría de discursos actuales consideran que la educación no solo se debe interesar por el desarrollo de conocimientos y habilidades, pues existe una necesidad de formación más profunda hallada en la naturaleza misma del ser.

En la tercera parte del artículo se brinda una justificación de por qué es necesario hablar de formación integral, dado que en el concepto de «formación» se evidencia un interés por desarrollar los aspectos humanos. La formación integral se presenta en las instituciones educativas como un estilo educativo que acentúa en las cualidades que permiten el crecimiento personal y social.

Las descripciones otorgadas acerca de la formación integral enuncian que el ser humano está constituido por dimensiones, pero no las concreta. En consecuencia, se presentan en el apartado cuatro las diferentes ideas que se han consolidado de la naturaleza del ser humano, y se concluye que las características de este se pueden agrupar en los aspectos bio-psico-sociales; esta condición requiere que en cada proyecto educativo se definan las dimensiones que se han de formar, de tal manera que se establezcan actividades que lo lleven a desarrollarse armónicamente.

En la quinta y última sección del artículo se reconoce en los fines de la educación superior el compromiso con la formación humana. El ser humano es un ser inacabado, por lo que tiene la capacidad de aprender a lo largo de la vida; esto justifica que las instituciones de educación superior planteen estrategias de formación orientada a sus diversas dimensiones, para que en la vida social actúe ética y responsablemente consigo mismo y con su contexto.

\section{EL INTERÉS DE LA EDUCACIÓN POR LA FORMACIÓN HUMANA EN LA ÉPOCA DE LA ILUSTRACIÓN}

En 1699 se conoce la obra pedagógica de Locke, titulada «Pensamientos sobre la Educación»; su ideario pedagógico contemplaba elementos de las ideas del Renacimiento, en las que se privilegiaba la cultura clásica (Locke, 1986), ahora complementadas con reflexiones sobre la crianza, los hábitos, la disciplina, las actividades lúdicas; pero sobre todo con el papel que debería cumplir la educación como proceso de consolidación de la razón humana. La razón, concebida no solo como la facultad con la que el ser humano está dotado por naturaleza, sino también como el proceso que, siguiendo un modelo matemático, permitía avanzar en la ruta del conocimiento y preparar al hombre para la vida social, es decir, formarlo para el ejercicio de la ciudadanía. 
Es de aclarar que, para Locke (1986), la educación tenía una connotación amplia que involucra todo el medio que puede influenciar al hombre; consideraba que el hombre ha nacido dotado de capacidades, facultades y potencialidades que pueden ser desarrolladas ampliamente solo a través del ejercicio, para convertirlas en habilidad y destreza, $y$ al igual que en el pensamiento posterior de Kant, estas características adquiridas pueden ser perfectibles, es decir, aptas para el desarrollo moral humano en toda su capacidad y complejidad. Tanto el cuerpo como el espíritu pueden ser ejercitados y conducidos; el uso recto de la razón es lo que le permite al hombre ser lo que es.

Entre otros aspectos, en Locke, la formación de los hábitos era fundamental para una buena educación; esto se logra a través de la disciplina, primero dirigida, y luego, conforme aumentan los años, se va otorgando más libertad. La educación, entonces, forma las costumbres y las actitudes; Locke la consideraba el único factor del progreso humano.

Para Locke, el interés de la educación debía estar centrado más en el desarrollo de las cualidades morales que en las intelectuales; el carácter moral permite constituir una sociedad de hombres con conciencia de su dignidad. Según esta idea, la razón guía, o debe guiar, la conducta del hombre, controlar sus apetitos, manejar sus inclinaciones y deseos, esto es, alcanzar el gobierno de sí mismo y lograr el sometimiento de la voluntad a la razón.

Un siglo después aparece Kant, quien, en sus lecciones sobre pedagogía, afirmaba que «el hombre es la única criatura que tiene que ser educada» (2009, p. 27); aclara que la educación comprende el cuidado del cuerpo, la disciplina que se brinda en los primeros años de vida, la instrucción y la formación. Como formación entiende el desarrollo espiritual, la orientación hacia la humanidad y su conservación, brindada en la crianza y la instrucción. Consideraba, además, que el hombre necesita de la crianza para ser humano, para ello debe hacer uso de la razón y de su propio esfuerzo. La crianza permite eliminar el salvajismo del hombre; la instrucción -de acuerdo con las ideas de Kant-es la herramienta para humanizar un ser que solo tiene la potencialidad moral, cualidad propiamente humana; la disciplina es vivir según las leyes de la humanidad, para ello es necesaria la coacción en el niño, para que cuando sea adulto tome decisiones libremente, pero guiado por su razón. 
Las aptitudes, según el pensamiento de Kant, son naturales en el hombre y tienen que ser desarrolladas; la educación debe estar orientada hacia el perfeccionamiento de la humanidad, por tanto, una verdadera educación será aquella que desarrolle en el hombre todas sus aptitudes naturales y que le permita alcanzar el fin de su existencia: la humanidad y la perfección. El hombre, por su parte, ha de aprender a sacar el bien de sí mismo, y a él mismo le corresponde desarrollar sus talentos.

Por tanto, para Kant la educación es indispensable para que el humano llegue a ser: disciplinado, para amansar su salvajismo; cultivado, a través de la enseñanza y la instrucción de sus habilidades; civilizado, lo que implica que sea inteligente, se adapte y se integre a la sociedad; moralizado, lo que implica la formación del espíritu; ilustrado, que aprenda a pensar, y no solo instruido mecánicamente. El hombre -a diferencia del animal-no es instintivo, por eso necesita de la educación. El arte de la educación es desarrollar la naturaleza humana, sus disposiciones para alcanzar su destino.

La finalidad de la educación en Kant es el cultivo general de las facultades anímicas, que son las mismas fuerzas inferiores del entendimiento; «facultad de conocer, de los sentidos, de la imaginación, de la memoria, de la fuerza, de la atención y del ingenio» (Kant, 2009, p. 85); así, comprende y recomienda cómo deben estas facultades ser desarrolladas con ciertas limitaciones en los niños. Asimismo, se deben cultivar las facultades superiores del entendimiento, el juicio y la razón, orientadas hacia el desarrollo de una razón que permita reflexionar acerca de lo que sucede, sus efectos y causas (Kant, 2009, p. 85). Y recomienda que la mejor manera de aprender y entender es aplicando lo que se está estudiando. Insiste que en la formación de la razón no es necesario que se les trasmitan a los niños conocimientos racionales, sino llevarlos a que ellos mismos los deduzcan.

En términos generales, Kant ve la educación como la herramienta que prepara para la vida, para el desarrollo personal y contribuye a la formación del hombre como ser social, para formar el carácter, el temperamento, la justicia, la moral, la razón, el esfuerzo, la disciplina, las habilidades, para el trabajo y la felicidad. La educación de la moral es necesaria para tomar decisiones sobre lo que es bueno o malo y sobre lo que más le conviene; formar el carácter, la capacidad de actuar bajo leyes, por lo que se necesita primero formar la obediencia y así podrá alcanzar su libertad como futuro ciudadano; a su vez, el 
hombre debe cuidar de sí mismo, esto es lo que comprende como dignidad de la humanidad, y a la escuela le corresponde formar el valor de la rectitud y de la honestidad.

Es así como el pensamiento de Locke, posiblemente, ejerció alguna influencia en el proyecto pedagógico de Kant, emblema del pensamiento de la Ilustración. La filosofía de la educación de Kant aún representa para la historia de la pedagogía un clásico, en la medida en que fundamenta una corriente de pensamiento orientada hacia el desarrollo de un ser más humano; toda vez que consideró la educación como la herramienta para formar hombres justos, libres y útiles para la sociedad. Las ideas de Kant acerca de formar al hombre desde su naturaleza permitieron comprender que la educación va más allá de trasmitir conocimientos y técnicas, por tanto, se constituye como el medio para desarrollar en el hombre su dimensión espiritual, moral, el pensamiento y las habilidades.

Así las cosas, la idea de «formación integral» se enriquece con la visión de estos dos filósofos de la Ilustración. Con estos legados, se da inicio a la consolidación de una corriente que estructura un pensamiento de la formación humana en todo acto educativo. Formar no es solo instruir, sino potenciar la «naturaleza humana» con miras a lograr un ser moral, racional, apto para vivir en sociedad y capaz de dirigir su conducta mediante la facultad de la razón, la cual debe gobernar y dirigir una vida plenamente humana en aras del perfeccionamiento moral. Estas características constituyen hoy la esencia de la formación integral, como se verá más adelante.

\section{UN ACERCAMIENTO AL CONCEPTO DE FORMACIÓN}

\section{El concepto de formación desde la tradición hermenéutica de Gadamer}

Con Kant y Locke se consolida una idea de las necesidades de educación del hombre para que llegue a ser humano, lo que implica un sujeto social con carácter; si bien sus escritos están centrados en la educación, el término específico de «formación» no es diferenciado, pues, al parecer, no existía la distinción. Gadamer (1960/1999), al intentar explicar el método en las ciencias del espíritu, realiza un recorrido preciso de la formación del hombre, condición básica para determinar el concepto de humanidad. 
Gadamer halla en Kant una génesis del significado de formación, cuando describe al hombre como un ser con disposiciones y talentos que deben cultivase y está en él mismo la obligación de hacerlo, dado que es el sujeto que actúa; entonces, es en Hegel donde encuentra un referente más preciso de la definición, a partir de esta idea kantiana, pues es el primero que habla de formación en este contexto y de formarse como un compromiso del individuo con sí mismo.

Para definir el origen de la palabra formación, el filósofo encuentra un concepto que se ajusta más al de formación humana: la palabra alemana Bildung -imagen imitada y modelo por imitar-, definida como la apropiación de la cultura influenciado por la tradición de su entorno, con una característica dinámica, de desarrollo y progresión constante.

Gadamer encuentra en Hegel que la formación es un concepto que conlleva un ascenso a la generalidad, «... acoge la determinación esencial de la racionalidad humana en su totalidad. La esencia general de la formación humana es convertirse en un ser espiritual general» (1960/1999, p. 15). Esta generalidad la explica como el sacrificio, la inhibición del deseo y de los propios intereses, por el bien de otros. Propone el ejemplo de la profesión; cuando alguien elige una profesión hace cosas que nunca hubiese hecho, que le son extrañas a su naturaleza; asume acciones externas, desconocidas y las apropia; se ocupa de cosas generales; la profesión se convierte en parte de su ser. Esta es la ascensión de su ser natural, hacia lo espiritual.

De estas ideas de Hegel, Gadamer concluye que el término de formación está vinculado a una tradición de su entorno cultural, pues recibe las condiciones del mundo exterior como el lenguaje, significados y costumbres, que pasan a ser parte de él. Y así, progresivamente se conduce hacia un ser espiritual general, esto es, desarrollo del interior; esta es la razón por la cual en sí mismo está la responsabilidad de darse forma o formarse.

En este sentido, la formación para las ciencias del espíritu se concibe como un concepto histórico, pues el hombre no es por naturaleza lo que debe ser, necesita formación de su esencia espiritual y racional. El ser va acumulando experiencias del mundo exterior y todo aquello que queda en él lo va transformando; todo aquello de lo cual se forma, no se olvida, hace parte ya de su ser. 
Si bien Hegel hace un aporte significativo para la interpretación de Gadamer, su pensamiento acerca del espíritu absoluto no lo considera, aunque la formación sea un elemento del espíritu. Lo que quiere decir Gadamer es que su postura sobre la formación y las ciencias del espíritu no toma todas las ideas hegelianas, ni se queda ahí. Por una parte, la formación acabada es un concepto que solo alcanza diversos desarrollos o madurezy, por tanto, lo deja en el ideal de las ciencias del espíritu. Por otra parte, el concepto de formación no puede ser únicamente ascenso a la generalidad, sino también es movimiento de quien se ha formado dentro de un elemento.

Entonces, Gadamer acude a Helmholtz y encuentra que el elemento de la formación es la sensibilidad o el tacto artístico, lo que le otorga una condición de libertad y movimiento al espíritu. La memoria es otro elemento como cualidad fundamental del ser histórico que ha de ser formada; lo retenido queda ambiguo, lo olvidado le permite al espíritu la renovación, la posibilidad de volver a observar y aprender, de relacionar con el recuerdo. Y finalmente propone la conciencia estética para poder juzgar y distinguir las cualidades de las cosas.

En conclusión, desde estas perspectivas, Gadamer fundamenta la concepción de la formación en la tradición hermenéutica; la describe como una acción que involucra el desarrollo de la condición humana y alude a esa necesidad de todo ser de avanzar y crecer desde su interior. Esto implica que al hablar de formación en una institución educativa se debe hacer referencia a algo más que una capacidad intelectual y técnica. Es necesario reconocer que es una idea ligada a lo que se concibe ser humano.

Gadamer concreta al ser con necesidad de desarrollo espiritual un ascenso a la generalidad, una disposición hacia lo otro y los otros, pero no con una meta última; lo que cobra sentido en el nivel de la educación superior. La característica histórica del ser permite que en su paso por esta experiencia educativa continúe su formación humana, se transforme en un ser con un papel determinado en la sociedad, según su profesión, y en esta construcción de ser profesional requiere de la apropiación de nuevos conocimientos, habilidades, destrezas y otras cualidades que alimenten su humanidad. 


\section{El concepto de formación entre el final del siglo XXY la primera década del siglo XXI}

Los autores de finales del siglo XX a inicios del XXI que presentan una propuesta del concepto de formación encuentran un asidero en las ideas de Gadamer frente a la necesidad del ser humano de desarrollar sus cualidades como ser, es decir, su humanidad, para ejercer un rol en el mundo como ser social. Ferry (1991) trabaja el concepto desde su preocupación por la formación de formadores; la describe como un proceso que permite el crecimiento y la perfección de capacidades individuales, tales como actuar, sentir, imaginar, comprender, aprender y utilizar el cuerpo; un hombre con formación será aquel que ha desarrollado sus características individuales para desenvolverse en el mundo.

De la misma manera, Remolina, Baena y Gaitán (2001) explican que la formación está ligada al concepto que se tiene del ser humano, de su composición interior; este está compuesto por cualidades que se pueden moldear, dar forma y conducir hacia un modelo de ser humano determinado. Para lograrlo, estos autores dicen que es necesario organizar sus estructuras, su interior, sus fundamentos como ser. Esta postura muestra la formación como una acción que cultiva el interior, el espíritu, el cual es susceptible de permanente construcción y transformación.

Remolina et al. (2001) presentan el concepto desde cinco elementos que lo conforman: el corazón, la sabiduría, la comunicación y capacidad de diálogo, los valores y la libertad. En el corazón se alojan y se cultivan sentimientos y afectos; desde allí se toman las decisiones importantes, y es la puerta al conocimiento. La sabiduría es el gusto por las cosas, permite desarrollar la capacidad de juzgar sensatamente el mundo, la historia, las personas y los hechos. La comunicación es el canal que hace posible el diálogo entre maestro y discípulo. La sensibilidad es la cualidad que se desarrolla para vivir en relación con otros. Y la libertad para actuar responsable consigo mismo y los demás. Esta descripción de Remolina contiene el interés de definir las cualidades básicas que han de ser formadas para lograr un ser humano que cuente con capacidades que le posibiliten vivir en sociedad; formación centrada en alimentar el espíritu, por lo que su definición se ubica dentro de la corriente de pensamiento de la educación para la humanización que propone Gadamer. 
Las ideas de Campo y Restrepo (1999) para conceptualizar la formación parten de lo que comprenden del ser humano; indican que por su naturaleza es un ser que está en devenir, en construcción, por lo que al pasar por la vida se irá desarrollando como individuo y su condición humana se constituirá según lo que él mismo haya nutrido; similar a lo que afirmaba Kant cuando decía que en la persona misma está la responsabilidad de cultivar sus cualidades. El desarrollo es, entonces, un despliegue de humanidad que está en permanente edificación y descubrimiento. De esta manera, Campo y Restrepo elevan la importancia para las instituciones educativas de reconocer la individualidad y, en coherencia, plantear acciones que lleven al ser a alcanzar su plenitud.

Hasta aquí, los autores mencionados proporcionan una idea de formación orientada al desarrollo de la persona; en este aspecto coinciden con las apreciaciones de Gadamer. Bajo esta perspectiva, los centros educativos que se comprometen con la formación de personas han de reconocer la naturaleza del ser humano y acompañarlo para que descubra sus potencialidades, pues, como ya se ha dicho, la formación no tiene límite. Bien se ha anunciado también en el informe de Delors (1996), que la educación es para toda la vida, y en este sentido la educación superior adquiere una tarea de continuar el proyecto humano.

\section{¿Por qué la formación integral?}

Si la formación se presenta como la acción encaminada hacia el desarrollo del ser desde su condición humana, ¿vale la pena agregarle la palabra integral? Esta adición ha sido criticada; hay autores que la consideran redundante, porque al hablar de formación, e incluso de educación, la integralidad está presente, ya que se parte del concepto de ser humano (Maya, 2003). Sin embargo, se considera necesaria la adición porque la educación o la formación que hoy día se brinda es diversa y, en ocasiones, especializada o tecnológica, lo que reduce los intereses en la formación. Esto abre la necesidad de acentuar lo humano con la palabra integral (Maya, 2003).

Vale aclarar lo que significa integral; en el DRAE se describe como «Dicho de cada una de las partes de un todo: Que entra en su composición sin serle esencial, de manera que el todo puede subsistir, aunque incompleto, sin ella» (2012). Es decir, que al entender la formación como un desarrollo del interior del ser, la integralidad se refiere a cada una de las dimensiones que componen a la persona. 
Campo y Restrepo (1999) aclaran que cuando se dice que la formación es integral se está hablando del sentido de esa formación. Se parte, entonces, de la visión que se tiene del ser, que está compuesto o integrado por dimensiones humanas; estas se encuentran, en constante interrelación y despliegue, presentes en todos los momentos de la existencia. En consecuencia, una educación que brinda formación integral deberá reconocer siempre dicha condición. En tal caso -afirman los autores- no habrá privilegios de la inteligencia sobre la afectividad del desarrollo individual sobre el social, ni se separará la imaginación de la acción.

El desarrollo armónico fundamenta la formación integral (Campo y Restrepo, 1999), que comprende al ser como una unidad en la diversidad. Esta unidad de dimensiones se desarrolla en el individuo de diferente manera, con diferente intensidad, en diversos tiempos. Así, la formación integral propicia el desarrollo del talento individual, para que cada quien sea lo que puede, viva y perfeccione sus fortalezas en el contexto de la totalidad de su ser.

El filósofo colombiano Luis Orozco (1999) desarrolla una idea parecida, pero la complementa; considera la formación integral como una práctica educativa centrada en los aspectos humanos, que permite crecer desde el interior en y para la libertad de cada quien, lo que contribuye a su proceso de socialización; indica que está relacionada con el desarrollo de su intelecto, su sensibilidad, su moral y su pensamiento crítico, para que, desde la autonomía y el potencial de su espíritu, logre comprometerse en la transformación de la sociedad en que vive. En esta postura de Orozco, la educación es integral si al estudiante le permite ir más allá de su potencial cognoscitivo o de la capacidad para el quehacer técnico o profesional. Coincide así Orozco con los conceptos anteriores, al afirmar que el ser debe ser reconocido como una totalidad integrada.

Asimismo, Rincón (1999) considera que la formación integral es un estilo educativo, donde se tratan saberes y elementos que permiten el desarrollo de los estudiantes como personas según sus singularidades; para que esto se dé, la educación debe formar en las dimensiones ética, espiritual, cognitiva, afectiva, comunicativa, estética, corporal y socio-político, de manera articulada. Aclara Rincón que al hablar de dimensiones no se busca separarlas en el proceso educativo, que es importante que las instituciones las discriminen para que comprendan al ser humano en formación y no dejar nada de su esencia por 
fuera, para garantizar su desarrollo pleno como persona (1999). Esta es la razón de acentuar la integralidad a la formación.

Zazar (2003) construye una propuesta orientada más hacia los aspectos de la formación en una educación integral y sus beneficios en el individuo, con base en tres autores: Bernard Lonergan (1993), Jerome Bruner (1915) y John Passmore (1914). Del pensamiento de Lonergan concreta tres aspectos fundamentales de la formación integral: el desarrollo intelectual para estimular la inteligencia y el desarrollo de juicio; los niveles de integración del conocimiento humano, los cuales la persona deberá ir alcanzando a través del desarrollo de la conciencia y del sentido común, con el fin de que se convierta en un ser activo y crítico dentro de su rol social, y la formación básica general, reconocida como el desarrollo de capacidades y habilidades intelectuales, lenguajes, actitudes y conocimientos de carácter humanista.

Con Bruner, Zazar describe que la formación integral busca el desarrollo del lenguaje para la comunicación, la adquisición de herramientas intelectuales, el desarrollo de la identidad y la autoestima, y así lograr que el alumno-término que usa Zazar-adquiera capacidades para desenvolverse en la sociedad de forma activa, crítica y transformadora. Estas capacidades son de tipo intelectual, físicas, procedimientos para trabajar, estudiar e investigar y actitudes, hábitos y valores.

A partir de Passmore, Zazar encuentra que una formación integral tiene la intención de desarrollar capacidades que le permitan al alumno adquirir información, cultivar hábitos de trabajo, para fomentar la imaginación, la actitud crítica, el interés por aprender y la capacidad de comprensión.

La síntesis que presenta Zazar, basado en los tres autores, es que la formación integral no debe descuidar ninguno de los siguientes aspectos, fundamentales para que la persona logre ser un sujeto activo, responsable con la sociedad y continúe su crecimiento personal y social en su continuar en la vida: manejo de información, desarrollo de habilidades intelectuales, destrezas físicas o motoras, métodos de investigación, hábitos, actitudes y la formación de valores para tomar decisiones y actuar de manera razonable, inteligente, responsable y amorosa. Esta propuesta destaca que la formación integral busca, a través del 
desarrollo de la persona, una preparación para la viday, por ende, un crecimiento para la sociedad.

Queda argumentado, entonces, que la formación por sí sola apunta hacia el desarrollo de los aspectos humanos y que la formación integral es un estilo o práctica educativa que comprende al humano como un ser compuesto por dimensiones; lo que implica que al asumir desde la misión institucional este tipo de formación, habrá un reconocimiento de las dimensiones del ser, para plantear estrategias pedagógicas que privilegien el desarrollo de todas.

Sin embargo, las descripciones otorgadas por los autores aquí referenciados no coinciden, ni determinan las mismas dimensiones; consideran que el ser es definido como un sujeto integrado y que esta integración ha de ser tenida en cuenta en el proceso formativo. Por lo tanto, en el siguiente apartado se presenta el concepto de ser humano y sus dimensiones, que han de ser tratadas en una formación integral.

\section{El SER HUMANO, SUS DIMENSIONES Y SU DESARROLLO}

Dado que el interés de la tesis y de este artículo es la formación del estudiante, el concepto de ser humano que se analiza aquí se orienta a buscar cuáles son las dimensiones de este, para comprender la condición de la persona que se forma en un proceso educativo. Por tanto, las concepciones de ser humano que se han ofrecido para el estudio de sus particularidades, como es el reconocimiento de sus origines, su cuerpo, su mente, su ciclo vital, su papel y desarrollo en la sociedad y su cultura, no se abordarán.

¿Qué es el ser humano?, es la pregunta que muchos se han planteado cómo problema filosófico a lo largo de la historia. Grandes pensadores, como Aristóteles, Santo Tomás y el mismo Kant, intentaron dar solución al cuestionamiento con una mirada desde la antropología filosófica, pero, según Buber (1949), no lograron hacerlo claramente, aunque sí brindaron una base fundamental para que otros filósofos trataran de dar respuesta, entre ellos Hegel, Marx, Feurbach, Nietzsche, Heidegger y Scheler.

Buber (1949) defiende la posición de Nietzsche, quien afirma que no es posible indicar cuál es la meta del hombre, pues, como él lo refiere, es solo un animal que promete, y estudia la individualidad de la persona en la colectividad, a 
través de la relación con el otro; en este estricto sentido, reconoce al ser dentro de la dualidad antropológica y sociológica que lo constituye, y encuentra que es un ser según sus posibilidades de relación, es decir, en comunidad y en recíproca presencia que le permite realizarse. Bajo esta visión del hombre, cobra sentido la necesidad de una permanente formación para que llegue a ser, gracias a la relación con el otro. No obstante, Buber, en búsqueda de dar respuesta al cuestionamiento ¿qué es el hombre?, que plantea en su obra, no se interesa por concretar las dimensiones que lo constituyen.

Se encuentra en Martínez (2009) un estudio que muestra que a mediados del siglo XX aparece una nueva concepción del hombre, basada en una visión integrada, no reduccionista y parcelada, como se pensaba hasta el momento. Una postura que comprende al hombre como un suprasistema, un conjunto dinámico, un todo integrado por dimensiones reconocidas como subsistemas coordinados: «el subsistema físico, el químico, el biológico, el psicológico, el social, el cultural, el ético-moral y el espiritual» (Martínez, 2009, p. 120). La integración de estos subsistemas conforma la personalidad. Dice el autor que la falta de integración o coordinación podría desencadenar problemas de tipo orgánico, psicológico o social.

Es difícil identificar los límites del cerebro humano, algunos autores indican que no se desarrolla plenamente; sin embargo, el desarrollo que logre depende de la formación adquirida en la sociedad y la familia; para Martínez, esto significa el despliegue de las estructuras físicas, químicas y biológicas en sentido estricto, y en sentido metafórico, la configuración de estructuras psíquicas, sociales, culturales, éticas y espirituales. Esto significa que como no hay una sola meta genética, se abren posibilidades de formación con orientaciones ideológicas diversas.

Bajo esta perspectiva, Martínez (2009) propone unas necesidades de desarrollo integral en el ser humano, dejando claro que existen otros enfoques que pueden complementar su propuesta; ellas son el desarrollo neurofisiológico; cognitivo, inteligencia y creatividad; psicológico, afectivo y social; moral, ético y de valores, y vocacional y profesional.

El desarrollo neurofisiológico (Martínez, 2009) está orientado hacia la satisfacción de la necesidad del ser humano de una buena alimentación y salud, como condición esencial para una buena estructuración del cerebro y el cuerpo; 
sobre esta condición favorable se podrá dar un adecuado desarrollo físico, químico y biológico.

El desarrollo cognitivo, inteligencia y creatividad (Martínez, 2009), se favorece gracias a un clima de libertad mental que valore el pensamiento divergente, autónomo y crítico. La inteligencia, razón y lógica, guía al hombre, aunque no siempre este la sigue cuando actúa; esto es porque su pensamiento está influenciado por la emoción y las afectividades que estimulan, desfiguran, inhiben o regulan el proceso cognitivo. La creatividad, como cualidad humana, es susceptible de ser estimulada por diversos factores del ambiente y del interior mismo de la persona, como la emotividad y la afectividad, por lo que se desarrolla en mayor o menor nivel.

El clima, afectividad y confianza, más un desarrollo físico sano, permiten el desarrollo psicológico, afectivo y social. El amor es la base fundamental para formar el carácter y lograr un desarrollo armónico, es el ingrediente que no puede faltar para una vida feliz. Dependiendo de estas condiciones se dará un desarrollo de las cualidades lingüísticas, intelectuales, emocionales, estéticas y éticas (Martínez, 2009).

El interés central de la educación humanista, para Martínez (2009), es el desarrollo moral, ético y de valores que parten de la unicidad del ser humano, de su individualidad y características propias que lo diferencian de los demás. Estos aspectos propios del ser hacen que cada uno adquiera una misión, unas metas y propósitos en la vida que se constituyen en la razón de su existencia.

Y, finalmente, la propuesta de Martínez se orienta hacia la necesidad de un desarrollo vocacional y profesional (Martínez, 2009); esto implica que los docentes busquen y generen problemas que involucren las dimensiones del ser: cuerpo, inteligencia y sensibilidad. Enfrentar a los estudiantes a situaciones en las que puedan desenvolverse con sus capacidades les permitirá crecer como seres humanos según sus posibilidades. Sobre estas ideas, se deberá cimentar la planeación de las actividades pedagógicas y educativas, y, así, acompañar la orientación vocacional de cada uno, de acuerdo con sus valores y cualidades personales, que le permitirán su desarrollo como ser.

Morín (1997), por su parte, presenta una propuesta que define al hombre como un ser biocultural; al interrelacionarse y mezclarse la cualidad biológica y la 
cultural que lo comprenden, se desemboca en esta doble proposición, ya que toda acción humana, como beber, dormir, comer cantar, aparearse, pensar, etc., es biológica y también cultural.

Morin (1999) complementa este pensamiento tiempo después, en su trabajo de La educación del futuro; dice que el hombre es un ser complejo, por su multidimensionalidad, definiéndolo como «a la vez biológico, psíquico, social, afectivo, racional» (1999, p. 16), por lo que este reconocimiento se hace necesario y fundamental para planear toda acción educativa, y agrega que existe gran dificultad, dado que esta condición humana permanece «atrozmente dividida», porque generalmente en las instituciones educativas no se concibe su unidad compleja. Esta situación se origina desde las ciencias humanas, que han permanecido fragmentadas. Por esta razón, Morin hace un llamado para guiar el futuro de la educación basado en las múltiples dimensiones del ser, como especie humana, como ser individual, social e histórico, y eliminar las divisiones, ya que todo conocimiento está interrelacionado con las dimensiones del hombre y se realimentan entre sí.

Morin (1999) entiende la complejidad humana en la relación de la triada individuo-sociedad-especie. Todo desarrollo humano lleva a un desarrollo de las autonomías individuales, de las participaciones en comunidad y del sentido de ser especie humana. La interacción entre individuos permite que exista la sociedad, sociedad que estructura la cultura, que tiene efecto sobre los individuos.

Genéticamente, la especie humana se caracteriza por su singularidad anatómica y fisiológica. Así, todo ser humano conlleva una unidad/diversidad de tipo cerebral, mental, psicológica, afectiva, intelectual y subjetiva. En este sentido, el humano está constituido bilógicamente por las mismas características, que a la vez hacen diferente y único a cada individuo. Entonces, comprender lo humano es comprender la unidad en la diversidad y la diversidad en la unidad (Morin, 1999).

En esta misma línea se presenta la idea de la unidad del ser, de Maya (2003); este la describe desde tres características básicas: bio-psico-social -cualidades que se asumieron en la tesis doctoral-. El «Yo» como totalidad es la integridad; esta es la respuesta que le da sentido a la formación integral y, por lo tanto, fundamenta la misión de educar a partir de un currículo que vele por el desarrollo 
de dicha integralidad, a través de programas, planes, estrategias y prácticas dentro y fuera del aula. Esta concepción bio-psico-social coincide con las reflexiones de Aristóteles: el hombre es un animal racional y un animal social o político.

Al afirmar Aristóteles que el hombre es un animal, se está haciendo referencia a su ser biológico, con necesidades básicas por satisfacer como cualquier especie animal con instintos para sobrevivir y reproducirse, por lo que busca alimentarse, refugiarse y autoprotegerse, entre otras cualidades que le permiten conservar su vida, su salud física y garantizar su existencia. En este grupo «Bio» podría ubicarse la dimensión corporal, la necesidad del conocimiento del cuerpo, su cuidado y el desarrollo.

La principal característica que diferencia al hombre de las demás especies animales es la capacidad de razonar; por esto, Aristóteles lo denominó animal racional. La condición humana es razón, inteligencia, intuición y sentimiento, que se expresa en la voluntad. El razonar le permite evolucionar y conformarse como un ser espiritual capaz de reflexionar y cavilar acerca de lo que lo rodea y de sí mismo. La concepción «Psico» hace referencia a la necesidad de un adecuado desarrollo psicológico del ser, de su mundo interior; involucra las dimensiones ética, espiritual, cognitiva, afectiva y estética.

Según la propuesta de Rincón (1999), la ética, como dimensión, se debe formar en la persona a partir de la libertad y autonomía para tomar decisiones desde su propia reflexión, dentro de los principios que rige la sociedad y la cultura en la que está inmerso -idea que coincide con el pensamiento de Kant-. La espiritualidad es la capacidad de trascender en su paso por el mundo. La cognitiva involucra el desarrollo de su capacidad intelectual para aprender, conocer y producir ideas, conocimientos. La afectiva brinda la posibilidad de amar y sentirse amado, de manejo de sus sentimientos y conflictos internos. $Y$ la estética desarrolla el concepto de belleza, el entender la vida y la naturaleza como un arte. Todos estos aspectos se construyen, se perfeccionan y solidifican a lo largo de la vida, es un proceso de continua construcción.

Finalmente, se puede relacionar la última expresión de Aristóteles, el hombre es un animal social, con la característica social de la triada bio-psico-social. Instintivamente, el ser humano busca establecer relaciones con los otros para distintos fines, y la comunicación es la herramienta que le permite establecer 
ese contacto. Por eso, cuando aprende a comunicarse, inicia su vida social. Esta categoría está relacionada con las dimensiones comunicativas y sociopolítica; involucra la necesidad de formar personas con habilidades comunicativas y políticas -no politiqueras-que se interesen por la estructuración y el bienestar de su comunidad.

Planteado este panorama conceptual del ser humano y del desarrollo de sus dimensiones, queda claro que la persona, durante su existencia, es susceptible de formación permanente, no solo de sus cualidades cognitivas, sino de todas las estructuras que lo componen como humano. En este orden, las instituciones de educación superior deberán asumir una postura acerca de la concepción humana, para que realmente se brinde una formación integral, a través de estrategias que permitan su crecimiento humano y cívico.

En este sentido, la educación superior deberá hacer un reconocimiento de la importancia que tienen los aspectos humanos en los procesos de formación profesional. El ideal es que el discurso docente rompa las barreras de la excesiva especialización y concentración en asuntos particulares del conocimiento y trascienda las estructuras humanas de cada estudiante. No se trata de que se formen profesionales o técnicos idénticos. Cada uno, con sus particularidades, podrá aportar de manera diferente a su área del saber, a la sociedad y al mundo, y llegar a ser, según sus potencialidades y capacidades innatas y adquiridas en el paso por la vida.

\section{LOS FINES DE LA EDUCACIÓN EN LA EDUCACIÓN SUPERIOR}

En la historia de las sociedades humanas, la educación ha estado presente como herramienta que permite desarrollar ciertas cualidades en las personas, según los intereses de los grupos líderes. Los espartanos, por ejemplo, necesitaban formar hombres para la guerra, y los atenienses, un sujeto político; desde estos fines, se planteaba un grupo de actividades y conocimientos que permitieran alcanzarlos (Hoyos, 2004); así, hasta los días presentes, ciertas sociedades o grupos establecen su propio pensamiento filosófico, sobre el cual plantean el proceso formativo. En el caso de Colombia, es fácil encontrar instituciones de carácter religioso, militar, técnico y científico, por mencionar algunos. 
Asimismo, a medida que el mundo cambia, los objetivos y fines de la educación se han ido replanteando. Sobre estas consideraciones, se afirma que siempre existirán unos intereses particulares que encaminan la formación de sujetos. Durkheim reconoció este dinamismo en los fines de la educación, afirmando que responden al contexto determinado por un grupo social, por lo que no existen unos fines ideales (Delval, 1990). No obstante, según los autores presentados a lo largo de este artículo, la formación de la condición humanaestados físicos, intelectuales y morales-deberá ser una constante en una promesa de formación integral.

Ya se explicó que el ser humano, por su naturaleza, requiere ser educado. Esta concepción humanizadora cimentó ideas de importantes pensadores - entre ellos los ya referenciados-que describieron los fines de la educación, encaminados esencialmente al desarrollo social e individual del ser humano. Pestalozzi y Ferriere-impulsores de una pedagogía activa-afirmaron que la educación de una persona no se puede alejar de la formación humana (2006).

La educación es la que convierte al ser humano en un integrante útil de la sociedad; así lo consideró Pestalozzi, en razón a que el desarrollo humano y cognitivo le permite alcanzar la autonomía y fortalecer su carácter moral. La acción formadora es condición necesaria para lograr seres que sirvan a una sociedad por sus saberes y por su libertad de pensamiento, en pro de consolidar una verdadera democracia. Para Pestalozzi, el fin moral es el fin supremo de la educación; sus ideas de educación expresaban una visión más integral de la que se reconocía en su época (siglos XVIII y XIX), visión que cultivará simultáneamente el corazón, la cabeza y la mano, lo que se acerca al concepto de formación integral. Este pensamiento lo continúa Dewey (inicios del siglo $\mathrm{xx}$ ), en busca de una pedagogía más humana y libre, alejada de los dogmatismos profesados por el poder eclesiástico.

En las reflexiones de Remolina (2001) se encuentra una propuesta como fin de la educación: la formación integral. Esta idea se complementa con la de Campo y Restrepo (1999), quienes afirman que cuando se habla de formación de personas se entenderá, de por sí, que es una concepción sobre lo humano. Si lo humano requiere de una formación integral, la educación está ante «su principal reto: saberse posibilitadora del proyecto de humanidad» (Campo y Restrepo, 1999, p. 4). 
La Unesco, en el año 1996, ya presentaba los pilares de toda educación, desde una visión integral, en razón a que no se concentra únicamente en una instrucción para un cúmulo de conocimientos: aprender a conocer, aprender a hacer, aprender a ser y aprender a vivir juntos (Delors, 1996). Desde este punto de vista, la educación se ha de preocupar por los aprendizajes del conocimiento y su desarrollo, del crecimiento como ser humano y sus relaciones con los otros, de tal forma que se aprovechen las posibilidades que el mundo ofrece.

En el mismo documento, Delors expone la misión de la educación, orientada a permitir que todos aviven sus talentos y capacidades de creación, para que cada ser se conozca a sí mismo, se responsabilice de sí mismo y genere su propio proyecto de vida, a partir del conocimiento, la autocrítica y la meditación. Así la educación girará en torno al desarrollo humano en todas sus dimensiones.

Para el caso particular de la educación superior, Delors (1996) explica que la misión es intelectual y social, para así garantizar los valores universales, el patrimonio cultural y el desarrollo de las naciones. Son las instituciones educativas las que deberán propiciar investigaciones para resolver problemas graves de su país y brindar formación profesional y técnica para disminuir las cifras de pobreza. Además, considera que en estos centros educativos se deberán discutir los problemas éticos y sociales de su país.

La Conferencia Mundial sobre Educación Superior también se pronuncia sobre la importancia de formar en la universidad con algo más que conocimientos en ciencia y técnica. Se destaca la responsabilidad de «contribuir además a la formación de ciudadanos dotados de principios éticos, comprometidos con la construcción de la paz, la defensa de los derechos humanos y los valores de la democracia» (Unesco, 2009, p. 2); con ello se deja claro el interés de formación que la educación superior debe brindar y el rol que se le delegada en la sociedad.

La misma idea defiende el filósofo colombiano Luis Orozco (1999), quien dice que la educación superior es el espacio donde se consolida el carácter, la personalidad, la ética y el pensamiento crítico del estudiante. Analiza Orozco que muchas veces se ha puesto en tela de juicio la responsabilidad de la universidad frente a los actos de sus egresados, por la fuerte influencia que en ellos se ejerció durante su proceso formativo. Solo es leer las noticias para reconocer que, en varias ocasiones, los profesionales son los protagonistas de la corrupción, que genera la violencia y la injusticia social. Es la institución de 
educación superior, en últimas, el espacio donde los estudiantes deben superar sus limitaciones frente al conocimiento y habilidades básicas, que impiden su continuary su formación. La universidad, finalmente, deberá estar comprometida con el desarrollo ético y crítico de los estudiantes.

Para Misas (2004), el propósito central de la educación superior es aumentar el conocimiento por medio de la investigación y transmitir saberes a través de la enseñanza; responsabilidad que se cumple gracias a la interacción entre «los actores del proceso de investigación y de aprendizaje, los medios materiales (edificios, laboratorios, bibliotecas) que están disponibles y la comunidad de valores y el clima social y cultural que brinde legitimidad y espacio a las actividades docentes e investigativas» (p. 239). Si bien este autor se centra en conocimiento e investigación, los pone en un escenario de aspectos humanos y sociales para alcanzar el fin, por lo que complementa su postura, añadiendo que la formación profesional está dada sobre la base de las dimensiones éticaestética-ciencia, tríptico que le permite al individuo desarrollar sus potencialidades y capacidades de creación.

Misas destaca la importancia del papel que juega la educación superior en el contexto nacional y regional, pues es aquí donde se cimienta el proyecto de desarrollo económico, social y político. En este sentido, estos centros deben contar con todas las condiciones necesarias para formar los futuros profesionales que sean capaces de comprometerse con la construcción de una nueva sociedad.

Es claro hasta aquí, a partir de los postulados de algunos autores, cómo se ha consolidado un pensamiento acerca de la educación para el desarrollo humano $\mathrm{y}$, por consiguiente, para una mejor sociedad. Tal vez, esta es la razón por la que diversos países adoptaron esta idea y la plasmaron en sus leyes, considerándola como uno de sus fines de la educación. Gutiérrez (2004) analizó los fines de la educación superior en algunos países miembros del ALCA, a partir de su legislación, y encontró que Ecuador, Perú, México, Guatemala, Chile, Argentina, El Salvador, Costa Rica, Honduras, Venezuela, Panamá, Bolivia, Nicaragua, Uruguay y Colombia consagran en su normatividad la formación integral como uno de los fines. En estos países se considera que la formación humana basada en el desarrollo moral y ético, combinada con la formación técnica, profesional y científica, hace de sus ciudadanos personas integras. 
Sin embargo, Ruiz (2002) hace una crítica acerca de la coherencia entre lo escrito y la realidad, pues a pesar de que la constitución mexicana -por mencionar un ejemplo-conciba la formación integral como su fin, las reglas de la globalización económica hacen que la educación superior desvíe su atención de los aspectos sociales y humanos y opte por una educación que prioriza «la información sobre la formación, la rentabilidad sobre la cobertura, el éxito individual sobre el beneficio colectivo» (Ruiz, 2002, p. 93). Esta es una realidad de los países inmersos en la dinámica económica implantada en Occidente, especialmente en los países en vías de desarrollo.

En el caso de Colombia, existe un marco normativo que apunta a la formación integral dentro de un concepto de desarrollo de las cualidades humanas. La Ley 115 de 1994, Ley General de Educación (1994), define que la educación es una formación permanente, fundamentada en un concepto integral de la persona; por consiguiente, establece que la formación integral «... física, psíquica, intelectual, moral, espiritual, social, afectiva, ética, cívica y demás valores humanos...» (Congreso de la República de Colombia, 1994, p. 2) es el primer fin de todo proceso educativo.

Algo semejante se propone desde la Ley 30, que define que la educación superior es «un proceso permanente que posibilita el desarrollo de las potencialidades del ser humano de una manera integral» (Congreso de Colombia, 1992, p. 1), y establece como primer objetivo «Profundizar en la formación integral de los colombianos dentro de las modalidades y calidades de la Educación Superior, capacitándolos para cumplir las funciones profesionales, investigativas y de servicio social que requiere el país» (Congreso de Colombia, 1992, p. 1).

El Consejo Nacional de Acreditación-CNA-(2013) también destaca el papel de la educación superior para el desarrollo integral de los colombianos; esta entidad evalúa las universidades que solicitan acreditación de alta calidad, en particular en aspectos como el currículo, si está orientado hacia la formación de valores, actitudes, aptitudes, conocimientos, capacidades y habilidades, y si existe un programa de bienestar y desarrollo humano, así como los espacios de participación de los estudiantes en actividades de formación integral, tales como «grupos o centros de estudio, en actividades artísticas, deportivas, proyectos de desarrollo empresarial-incluida la investigación aplicada y la innovación-, y 
en otras de formación complementaria, en un ambiente académico propicio para la formación integral» (Consejo Nacional de Acreditación, 2013, p. 23).

Este marco se expresa en políticas, estrategias y planes que las universidades colombianas deben desarrollar para el logro de los objetivos del sistema educativo, incluso, se puede afirmar que la formación integral es un aspecto visible en los fundamentos filosóficos de toda institución de educación superior. Las estrategias más comunes que se plantean y que el mismo CNA exige para las universidades que buscan la calidad son la inclusión de asignaturas orientadas a desarrollar la dimensión ética, estética, ambiental, filosófica, política y social, y de una política de bienestar universitario que propicie un clima para el desarrollo humano (Consejo Nacional de Acreditación, 2013).

Queda, entonces, afirmar que la formación integral sí es el fin de la educación superior; no obstante, como indica Orozco, el problema «es que habiendo una posición tomada sobre el tema, expresada en los estatutos de las instituciones de educación superior (IES), con mucha frecuencia no es más que un reconocimiento retórico con poca incidencia práctica» (2011). Un reflejo de esta situación se expresa en la forma como las instituciones de educación superior en Colombia organizan la formación del estudiante; se delega a diferentes departamentos la responsabilidad de brindar desarrollo de una parte de sus dimensiones, lo que ocasiona que los mismos docentes y estudiantes otorguen más importancia a unas actividades que a otras. Sumado a este escenario, existe una presión de la economía global, que demanda un profesional «competitivo» con altas capacidades específicas en una ciencia o saber, por lo que estos centros educativos se concentran en formar profesionales para el desarrollo económico de sus países.

Se halla en la realidad, que en la formación de los profesionales hay un acento especial en el aprendizaje y desarrollo de conceptos, teorías y habilidades. Los aspectos humanos, que requiere la formación integral, son de gran relevancia en los proyectos educativos universitarios (Nova, 2013), pero con reducidos compromisos de quienes educan. Nussbaum ha presentado esta crítica: «actualmente predomina una nueva concepción en torno de la rentabilidad y que ha dejado de lado la idea del desarrollo personal basado en la imaginación y en el pensamiento crítico» (2010, p. 22), lo que ha ocasionado un desprecio por las humanidades, las artes y la filosofia. 
Bajo esta perspectiva y ante tantos conflictos sociales, vale la pena hacer un llamado a la reflexión para recobrar el papel de la educación superior frente a la formación de la condición humana, para que la realidad muestre un panorama donde el estudiante y el docente reconozcan que la esencia de toda educación ha sido y seguirá siendo llevar al ser a su máximo desarrollo como ser humano, no importa la edad o etapa de la vida en que se encuentre, para construir una sociedad de ciudadanos pacíficos y con menos frustraciones.

\section{Conclusiones}

La educación ha sido vista a lo largo de la historia de la sociedad occidental como un instrumento esencial para la formación del ser humano, que ha sido considerado, a diferencia de los animales, como un ser indeterminado que necesita orientación para que llegue a ser lo que debe ser como humano. Esta afirmación requiere que las instituciones de educación, y en especial de educación superior, comprendan al ser humano con sus características biológicas, psicológicas y sociales, dotadas de dimensiones que necesitan ser potenciadas según la diversidad de cada individuo, para garantizar ciudadanos comprometidos con su propia vida y con la construcción de una mejor sociedad.

Se ha considerado, erróneamente, que la educación en los aspectos humanos que forman al ser según sus individualidades es una tarea principalmente de la educación preescolar, básica y media, y de las familias en las primeras etapas. Si bien es allí donde de forma principalmente el carácter, esta naturaleza indeterminada del hombre permite a las instituciones de educación superior alimentar el espíritu de sus estudiantes y fortalecer sus habilidades para relacionarse con los otros, para que logren transformarse en los profesionales que eligieron ser.

En este sentido, velar por la unidad de la especie humana sin eliminar su diversidad, como lo mencionaron Morin y Campo y Restrepo, delega a las instituciones de educación superior el reto de replantear la formación desde sus dimensiones. Esta condición está siendo tratada y formada por diferentes departamentos o áreas, tales como el de humanidades, de biología, de literatura, de filosofía o el bienestar universitario. Esto ratifica la situación que plantea Morin, al afirmar que los problemas del mundo están siendo analizados desde departamentos o áreas específicas. El hombre formado por disciplinas 
fragmentadas corre el riesgo de no contextualizar lo global y natural, lo que le otorga una visión reducida del mundo y lleva a que cada ser se responsabilice por solucionar una parte de cada problema.

Se destaca el marco normativo de Colombia para la formación integral, especialmente la Ley 30 de 1992 de educación superior, la cual exalta la importancia de formar personas desde su condición integral. Asimismo, la coherencia de este discurso con el de las universidades, que desde sus proyectos educativos plantean estrategias y ofrecen currículos que contienen diversos componentes humanísticos, que apuntan no solo a la formación específica en un área o ciencia determinada, sino que brindan la oportunidad de desarrollar sus dimensiones. Sin embargo, es lamentable que se robe toda la atención de la comunidad académica formar para el trabajo o para el crecimiento económico, y no se comprenda que la ciencia, la tecnología, los negocios y demás actividades provenientes de la acción profesional necesitan sujetos éticos, ciudadanos justos que generen condiciones de vida equitativas para todos, felices, que construyan un proyecto de vida no solo al servicio del mundo, sino también de su propio bienestar a través del fortalecimiento de sus habilidades y capacidades individuales.

\section{REFERENCIAS}

Buber, M. (1949). ¿Qué es el hombre? México: Fondo de Cultura Económica.

Campo, R. y Restrepo, M. (1999). Formación Integral: Modalidad de educación posibilitadora. Santafé de Bogotá: Pontificia Universidad Javeriana.

Congreso de la República de Colombia (1992). Ley 30: por la cual se organiza el servicio público de la Educación Superior. Recuperdo de: http:// www.mineducacion.gov.co/sistemasdeinformacion/1735/articles211884_Ley_30.pdf.

Congreso de la República de Colombia (1994). Ley general de educación 115. Recuperado de: http://www.mineducacion.gov.co/1621/articles85906_archivo_pdf.pdf 
Consejo Nacional de Acreditación (2013). Lineamientos para la acreditación de programas de pregrado. Recuperado de: http://www.cna.gov.co/ 1741/articles-186359_pregrado_2013.pdf

Delors, J. (1996). La educación encierra un tesoro. París: SantillanaUNESCO.

Delval, J. (1990). Los fines de la educación. España: Siglo XXI.

Echeverría, B. (2010). Orientación profesional. Barcelona: UOC.

Ferry, G. (1991). El trayecto de la formación: los enseñantes entre la teoría y la práctica. México: Paidós.

Gadamer, H. (1960/1999). La superación de la dimensión estética. En H. Gadamer, Verdad y Método I (A. Aparicio y R. de Agapito, Trads., pp. 31-74). Salamanca, España: Sígueme.

Gutiérrez, H. (2004). Fines de la educación superior en la legislación de algunos paises miembros del ALCA. Recuperado de http:// www.mineducacion.gov.co/1621/article-85906.html.

Hoyos, S., Hoyos, P., y Cabas, H. (2004). Currículo y planeación educativa. Bogotá: Actualización Pedagógica Ministerio.

Huanacuni, F. (2010). Buen vivir/Vivir bien. Filosofía, políticas, estrategias y experiencias. Coordinadora Andina de Organizaciones Indígenas CAOI-. Recuperdo de: https://www.reflectiongroup.org/stuff/vivir-bien

Kant, I. (2009). Sobre Pedagogía. Coìrdoba: Encuentro Grupo Editor.

Locke, J. (1986). Pensamientos sobre la educación. Madrid:

Locke. (1992). La conducta del entendimiento y otros ensayos póstumos. Barcelona: Anthropos.

Martínez, M. (2009). Dimensiones básicas de un desarrollo humano integral. Revista de la Universidad Bolivariana, 8(23), 119-138. 
Maya, A. (2003). Conceptos básicos para una pedagogía de la ternura. Bogotá: ECOE.

Misas, G. (2004). Colombia aprende. La educación superior en Colombia: Análisis y estrategias para su desarrollo. Recuperado de: http:// www.colombiaaprende.edu.co/html/investigadores/1609/articles73081_a

Morin, E. (1997). La unidualidad del hombre. Gazeta de Antropología.

Morin, E. (1999). Los siete saberes necesarios para la educación del futuro. París: UNESCO.

Nova, A. (2013). Políticas institucionales que favorecen la formación integral en la educación superior colombiana. Heuristica: revista digital de historia de la educación, 16, 82-95. Recuperado de: http:// www.saber.ula.ve/bitstream/123456789/37847/1/art5.pdf

Nussbaum, M. (2010). Sin fines de lucro. Buenos Aires: Katz editores.

Orozco, L. (1999). La formación integral: Mito y realidad. Bogotá: Ediciones Uniandes.

Orozco, L. (2011). La formación integral: Menos retórica y más reconocimiento de la tarea necesaria. Universidad y Cambio. Recuperado de: http:// universidadycambiosocial.blogspot.mx/2011/03/la-formacion-integralmenos-retorica-y.html

Pestalozzi, J. (2006). Cartas sobre educación infantil. Madrid: Tecnos.

Real Academia Española (2012). Diccionario de la lengua española. Recuperado de: http://buscon.rae.es/draeI/ SrvltGUIBusUsual?LEMA=integral

Remolina, G., Baena, G. y Gaitán, C. (2001). Tres palabras sobre formación. Bogotá: Pontificia Universidad Javeriana.

Rincón, L. (1999). La formación integral y sus dimensiones: Documento de trabajo. ACODES.I Recuperado de: http://www.ipatria.edu.mx/ 
descargas/LA_FORMACION_INTEGRAL_Y_SUS_DIM ENSIONES_TEXTO_DIDACTICO.pdf

Ruiz,A. (2002). Educación Superior y Globalización. México: Plaza y Valdéz.

UNESCO (8 de julio de 2009). Conferencia Mundial de educación Superior: La nueva dinámica de la educación superior y la investigación. Recuperado de http://www.unesco.org/education/WCHE2009/ comunicado_es.pdf

Zazar, C. (2003). La formación integral del alumno: qué es y cómo propiciarla. México: Fondo de Cultura Económica, colección Educación y Pedagogía. 